\title{
Maximizing the Information Diffusion Opportunity in the Cyber-Physical Network
}

\author{
Hongliang Lu*, Xuan Dong*, Wenxiang $\mathrm{Li}^{\dagger}$, Saohe $\mathrm{Lv}^{*}$, Xiaodong Wang* and Wei Chen ${ }^{\S}$ \\ ${ }^{*}$ Science and Technology on Parallel and Distributed Processing Laboratory, \\ National University of Defense Technology, Hunan, China. \\ Email: \{honglianglu, dongxuan, shaohelv, xdwang\}@nudt.edu.cn \\ ${ }^{\dagger}$ School of Information Science and Engineering, Wuhan University of Science and Technology, \\ Wuhan, China. Email: \{liwx2006\}@ hotmail.com \\ $\ddagger$ Engineering Research Center for Metallurgical Automation and Detecting Technology of Ministry of Education, \\ Wuhan University of Science and Technology, Wuhan, China. \\ $\S$ College of Computer Science and Technology, China University of Mining and Technology, \\ Xuzhou, Jiangsu, 221116, China. Email: \{chenw\}@cumt.edu.cn
}

\begin{abstract}
Our daily life is changing by the smart objects, such as smart watches, smart phones etc. They make the cyber world and the physical world integrated by their abundant abilities of sensing, communication and computation etc. Focusing on a wide range of the integrated network, a statistical based strategy was introduced to get a special kind of link between objects, the statistical probability communication link. To get a maximized information spread probability for grouped people, this paper introduced a distributed, yet efficient algorithm naming DMPID algorithm, for finding a sub-network to spread people oriented inforamtion. The DMPID algorithm take the size of the selection and the information spread probability into account, and made a balance between the two parameters. Extended simulation showed that the DMPID algorithm performs well in different distributed networks.
\end{abstract}

\section{INTRODUCTION}

Benefit from the advances in sensor networks, the cyber physical network appeared as a innovation technology to integrate the cyber-space with the physical-space. It makes the mobile computing, smart sensing and smart controlling more sophisticated. It's expected that in the future, the integration of cyber space and physical space can make our daily lives easy and efficient. By connecting the daily objects, such as television, car, refrigerator, laptop, even coffee cup, key etc. these objects can exchange information between each other, and such form a heterogeneous network. This network can also co-related with the users' cyber social networks. An example of such correlation network is shown in Fig. 1. Compared with the traditional internet that focuses on the interconnection of computers, the cyber physical network builds a wider network that consists of both the computers and various physical objects in the world. The Cyber-Physical network is widely used in many fields such as green computing, intelligent power service, smart city and others. It is also regarded as an important data source and application area of big data technology. In the cyber physical network, there exist various kinds of relations among the physical and cyber objects (as shown in Fig. 1). The relations can be described in different dimensions, such as spatial dimension, temporal dimension,

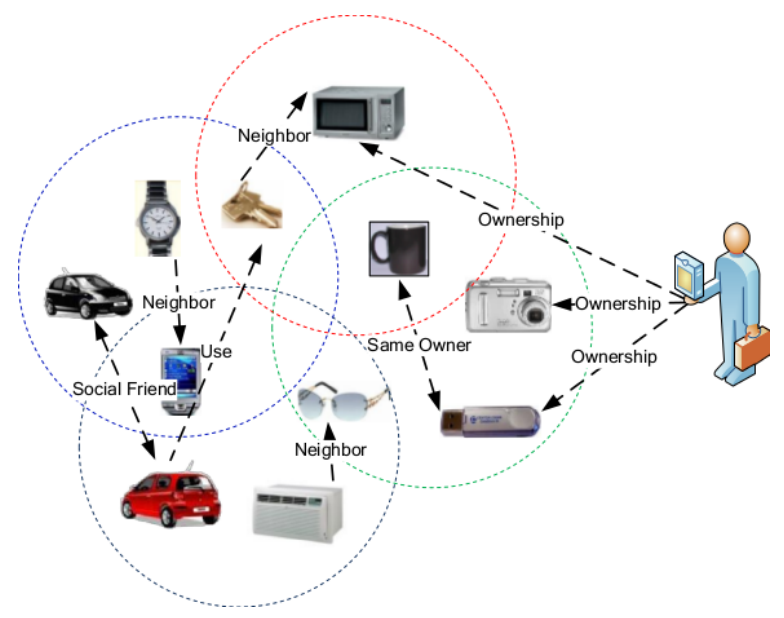

Fig. 1: An example of cyber-physical network

social dimension and others. For example, neighbor relation is a common relation that described in spatial dimension, which may refer to that a key resides near a laptop, or a watch lies on a television. And co-owner relation is a common relation that described in social dimension, which may refer to that a car and a camera, or a USB storage device belong to a common owner. Besides, friend relation is a widely used ubiquitous relation, which may refer to that the owner of two cars are friend in the cyber space. The relations are important for the user to extract needed knowledge and navigate among objects. Considering that cyber physical network usually related to a large number of objects, the extracted relations will in large volume and can be feeded into big data tools for further processing.

Basing on the relations mentioned above, different types of interesting applications can be taken out. Such as accurate information flooding, some information is needed to be forwarded to a certain group of people for example, people oriented advertisement, urgent temporary cooperation, take emergency care as an example, etc. However, these applica- 
tions need the participant to cooperation in higher opportunity . The task to choose the crowd is quite challenging, because these cyber-physical objects have been changing all the time. And the relation between the objects is changing all through time. The main difficulty is that a global snapshot of the network is usually needed to analyze the relations between objects so as they can be selected as the collaborators. In these cases, the key problem is not to deal with the communication problems in wireless network, but to ensure the opportunity of the cooperation so as to finish the tasks. As a result, when choosing the collaborators, the priority criteria is the opportunity that the objects can communicate between each other.

Existing works on cyber physical relations focuses on how to find an efficient algorithm to collect the needed information. Not considering that besides the algorithm, the dynamic of the network is the most important factor in the cyber physical environment. In previous works [1], [2], it is focuses on collecting the information from a collection of objects to infer the relations. They take a centralized strategy, which collect the information from the distributed objects to one centralized object. This introduces several intuitional problems, such as one-point failure problem, finding the strong enough server is difficult, and in cyber physical network, such centralized structure introduces serious security risks. Therefore, a suitable strategy is needed to support applications demands in these cyber physical network, so as to meet these opportunity dependent tasks in such environment.

In this paper, we propose a distributed algorithm to find and construct a sub-network, which has the higher probability, meanwhile, keep a good network characteristic. Basing on this sub-network, we can deploy different types of algorithms for the cyber physical applications. We first analyze the characteristics of the cyber physical network and make up the distributed system model for this problem. After that, we illustrate our algorithm how to construct the sub-network for information diffusion. The algorithm fully used the neighbor information and optimized for the opportunity connections between objects. Extensive simulations are carried out to validate the effectiveness of the proposed algorithm. The results show that the proposed approach is effective and outperform the existing approaches. In summary, this paper makes the following contributions.

- We build a distributed system model for the cyber physical network to support different types of applications.

- We proposed a distributed algorithm to make a selection in the cyber physical environment so as the information diffusion in a higher probability.

- We conducted extensive simulations to validate the effectiveness of the proposed algorithm.

The rest of the paper is organized as follows: Section II describes the system models used in this paper. After that, the distributed sub-network construct algorithm is illustrated in Section III. The simulation results for the proposed algorithm are reported in Section IV. Section V reviews the related works and finally Section VI concludes the paper.

\section{System Model AND Problem Formulation}

Co-relation of the cyber network and the physical network, forming a more complex network. It's heterogeneous both in the nodes and links. We call such a network cyber physical integrated network. The core characteristic of such a network is dynamic. Which means that the nodes and the connection between nodes in the network is changing all through the time.

\section{A. Integrated Network Model}

The cyber physical integrated network has the characteristics similar as that in sensor networks, objects can communicate with other ones which lies nearby. And whenever they lies in the data transmission range with each other, they can not directly bi-communicate. We model the integrated network as a bidirectional graph $G=(V, E)$, where $V$ is the objects set and $E$ is the communication channel set. The links are setup when they satisfy the following constraints: $\forall u, v \in V$, there exists a edge $E(u, v)$ between $u$ and $v$, if and only if:

- $u$ lies in the $v$ 's transmission range.

- $v$ lies in the $u$ 's transmission range.

- there is no obstacle that can prevent electronic transmission between $u$ and $v$.

However, in the cyber physical integrated network, the communication channel between objects is not in a constant status so long as they may moving or moved time to time. The communication between two objects is made when they are in the data transmission range within each other. This is happened in an opportunistic manner within a period of time. We introduce the probability to describe the communication opportunity.

In the cyber physical integrated network, each object $v_{i}$ has a probability connection $P_{i j}$ to object $v_{j}$. The probability can be derived from the statistical characteristics of the network, and even more, the probability is different at different time of a day. So the cyber physical integrated network is modelled as a graph $G=(V, E)$, where $V=\left\{v_{1}, v_{2}, \cdots, v_{n}\right\}$ corresponding to the objects, and $E$ is the set of communication links between any pair of objects. Each $e_{i j} \in E$ has a attribute of probability $P_{e_{i j}}$.

In this paper, we focus on find the stable sub-network for a certain time in a day to maximize the probability of information diffusion. So the objective is to construct a sub-network from the network described above, so as the information can spread in a higher probability. However, respect to the concept of lifetime in network, the probability is determined by the minimum communication link of the network. A example network shows in Fig. 2, in which the maximum information diffusion probability is 0.4 .

\section{B. Problem Formulation}

As described in part I, we want to maximize the information diffusion opportunity in the cyber physical integrated network. The critical task is to find a sub-network, so that objects can transmit the information to their neighbor in a higher 


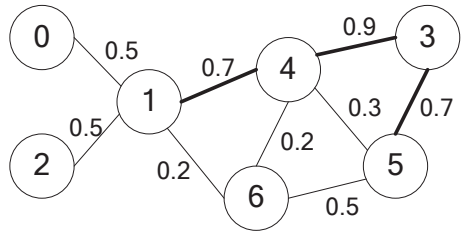

Fig. 2: An example network

opportunity. At the same time, the objects selected for the information spread is minimized. Formal description as:

Maximizing Probability of Information Diffusion(MPID). Given a network $G=(V, E)$ and the distribution of the nodes, the MPID problem is to find a minimum connected sub-set $S$ to meet the following demands:

- the size of the sub-set $S$ is minimized, $\min \{|S|\}$.

- the probability for information diffusion through the subset is maximized, $\max \{\operatorname{Prob}(S)\}$

The MPID problem is a multi-objective optimization problem. And the two objective can not be optimal at the same time. To let the information spread all over the network, the sub-network should dominate all other objects in the network. At the same time these selected objects should keep a high connection probability. For the cyber physical integrated network is a dynamic network, and the probability connection is different time to time, so the valid of the links in the network should be taken into consideration. In this paper, we assume that the information diffusion time is much less than the time for constructing of the sub-network.

\section{Algorithm Design AND ANALysis}

The cyber physical integrated network is formulated to be a bidirectional graph, and the algorithm is designed based on the neighbor information. The prerequisites is that the information of neighbor and two hops neigbor have been collected.

\section{A. Distributed MPID Algorithm}

In the cyber physical integrated network, constructing a subnetwork that can communicate with all the other objects is an almost impossible task. Specially, a centralized method is completely not applicable. So in such a network, a distributed and low message complexity algorithm is desired. Inspired by the distributed algorithms in wireless sensor network [3], we adopt the two hops information based algorithm design strategy.

For convenience, we define the dominant ability taking the size of the selection and information spread probability into account. Given a node $v$, and his neighbor $N(v)$. The dominant ability of $v$ for his neighbor is defined as

$$
D A(v)=\alpha * a m(v) *|L N(v)|+\beta * C N(v)
$$

In the equation, $\alpha$ and $\beta$ is the non negative coefficient of the two elements, $\operatorname{am}(v)$ is the arithmetic mean of the probability of the links. i.e

$$
\operatorname{am}(v)=\operatorname{mean}\left\{P_{v, u} \mid u \in N(v)\right\}
$$

The $L N(v)$ is the links that has a higher probability than the $\operatorname{am}(v)$

$$
L N(v)=\left\{u \mid P_{u, v}>a m(v), u \in N(v)\right\}
$$

And the $C N(v)$ is introduced to describe the link with the minimum probability.

$$
C N(v)=\left|\operatorname{am}(v)-\min \left\{P_{v, u} \mid u \in N(v)\right\}\right|
$$

The definition of the $a m(v)$ and $|L N(v)|$ take both the probability and the size of the links into account, while $C N(v)$ is deserved for considering the link with lowest communication probability. Since the probability of the links is a statistical parameter for a certain time range, the $a m(v)$ can be respected to contribute for maximizing the probability of the final sub-network, and the $|L N(v)|$ is to minimizing the size of the selected nodes, while the $C N(v)$ is balancing the two objectives. Intuitively, nodes with more neighbor and have higher communication probability will play more important role in hop by hop communication.

Besides, in some cases, one node and his neighbor can be dominated by another node. In that case, the node can be pruned from the selected sub-network, so as to reduce the size of the final sub-network. The definition for describing the ability for a node to dominate a set of other nodes as:

$$
D A S(\{v\}, S)=\alpha * \operatorname{ams}(v) *|L S(v)|+\beta * C S(v)
$$

In equation 5, the $\operatorname{ams}(v)=\operatorname{mean}\left\{P_{v, u} \mid \exists e_{u, v}, u \in S\right\}$, denotes the arithmetic average probability of the links, through which the node $v$ can connect to the nodes in set $S$. The $L S(v)=\left\{u \mid P_{u, v}>\operatorname{ams}\left(P_{v, u}\right), u \in S\right\}$, is the number of the links that has a higher communication probability compared with the average probability. The $C S(v)=$ $\left|\operatorname{ams}(v)-\min \left\{P_{v, u} \mid u \in S\right\}\right|$, is the distance for the lowest communication probability to the average probability. And the $\alpha$ and $\beta$ is coefficient for these two element. The equation represents the ability of node $v$ dominate the nodes in set $S$.

In some cases, a node and his neighbor may be dominated by his multiple neighbor. Thus, even the node is pruned from the selection, the node and his neighbor can participant the information diffussion either. The definition for the dominant ability of set to set as:

$$
D A S\left(S_{1}, S_{2}\right)=\alpha * \operatorname{ams}\left(S_{1}\right) *\left|L S\left(S_{1}\right)\right|+\beta * C S\left(S_{1}\right)
$$

In equation 6, the $\operatorname{ams}\left(S_{1}\right)=\operatorname{mean}\left\{\max \left\{P_{u, v} \mid u \in\right.\right.$ $\left.\left.S_{1}\right\} \mid v \in S_{2}\right\}$ meaning that given a node $u$ in set $S_{1}$, choose the link (through which $u$ connected to set $S_{2}$ ) with the highest probability, then get the arithmetic average of these probability. And the $L S\left(S_{1}\right)=\left\{e_{u, v} \mid u \in\right.$ $\left.S_{1}, v \in S_{2}, P_{e_{u, v}}>\operatorname{ams}\left(S_{1}\right)\right\}$ is reflecting the number of links, through which the two set are connected. $C S\left(S_{1}\right)=$ $\left|\operatorname{ams}\left(S_{1}\right)-\min \left\{\max \left\{P_{u, v} \mid u \in S_{1}\right\} \mid v \in S_{2}\right\}\right|$ describing the distance from the lowest probability of the links to the average probability. The equation represent the ability for nodes in $S_{1}$ dominate the nodes in $S_{2}$. This is used to prune the initial selection made by intuitive strategy. 
Similar as that most distribute algorithms, we design the Distributed MPID algorithm (DMPID) as a three stage algorithm. The first stage finishes the tasks for collecting neighbor information and the initial role of the nodes in the network. To be specific, each node collect his one hop and two hops neighbor information through data transmission. Using these collected information, node $v$ determines his initial role. If the two node $u$ and $w$ both are the neighbor of node $v$, and there is no direct link between them, i.e the link $e_{u, w}$ dose not exist, the node $v$ can be determined to be a dominator, and should be marked as Blue.

The sencond stage of the DMPID algorithm is to reduce the initial selection. A dominator selected in the first stage will be pruned from the selection and marked as Green through the following two criterions:

- a dominator and his neighbor can be dominated by one of his neighbor, then the dominator can be pruned.

- a dominator and his neighbor can be dominated by a set of his neighbor, then the dominator can be pruned.

Through the above two criterions, the selection of the nodes is reduced. But in some cases, this is not good for maximizing the communicatipon probability. For example, in Fig. 2 the node 5 will be pruned from the selection after the sencond stage. In fact, if introduce the node 3 as an alternative, the communication probability of the selection is much higher. So the stage three of the DMPID algorithm is to check these one hop connections to the selection from the candidates to be pruned. Take the node $v$ that has to be pruned as an example, if there exist a node $u$ in the neighbor of $v$, $\exists u \in N(v)$, at the same time $u \in N(w)$, where $w$ is a dominator, and $\min \left\{P_{u, w}, P_{u, v}\right\}>P_{w, v}$, we define the profit as the increment of the two links compared to the original link, refer the equation 7 .

$$
\operatorname{Pro}(v, u \mid w)=\frac{\min \left\{P_{u, w}, P_{u, v}\right\}-P_{w, v}}{P_{w, v}} \times 100 \%
$$

If the profit achieves a threshold $\gamma$, then the node $u$ is added as an additional selection and node $v$ avoided to be pruned. The pseudo code of the DMPID algorithm is listed in algorithm 1.

\section{B. Analysis}

The distributed algorithm based on the neighbor and two hops neighbor information has been proofed to be valid and correct[3]. We take a similiar strategy to design the DMPID algorithm, and every node in the network make decision based on the neighbor and two hops neighbor information. From the perspective of each node, it's totally using the local information. Here, we briefly analyses the correctness, valid and complexity of the DMPID algorithm.

As stated in the system model, in the cyber physical integrated network, the objects can communicate with each other in a probability manner. Given a time, the communication probability can be determined based on statistical and predicate methods. During a certain time period, the application related objects can form a connected network. In

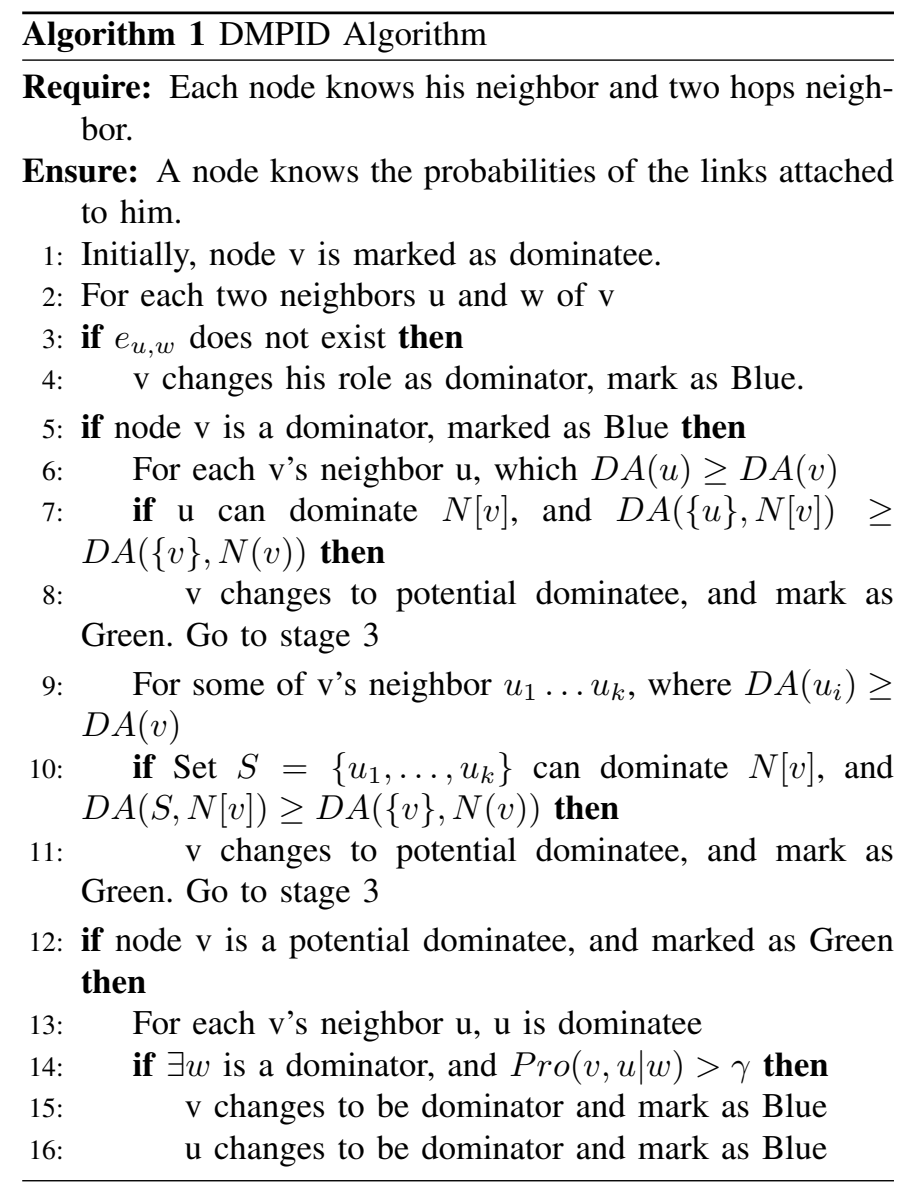

the first stage of the DMPID algorithm, a node is chose as a dominator if the node has two neighbor, but the two nodes has no chance to communication with each other. Suppose node $v$ and $u$ are two nodes in the network, and they have no chance to communicate, in other words, there is no link between them in the network. But the network is a connected network, through some hops node $v$ can reach to node $u$. The simplest situation is through two hops, thus as the algorithm shows, the intermediate node $w$ will be chose as dominator. In such a scenario, any node that link to the node $w$, if he is not linked to $v$ or $u$, he will be selected as dominator. In this way, the nodes in the network chosen as dominator is connected. And if the path from $v$ to $u$ is longer than two hops, then there exist a node on the path that is two hops to $v$. Thus, it's the same as that of the situation ahead. Therefore, in the algorithm stage 1, a connected sub-network will be selected. In stage 2 and 3 , operation is performed on the premise of guaranteeing the connectivity. As a result, the selected subnetwork will keep connectivity all through the algorithm. It shows that the DMPID algorithm is valid.

After the first stage of the DMPID algorithm, a connected sub-network is selected.A pruning process is needed to reduce the size of the selection. The criterions for reducing the size of the selection is a node can be replaced by other nodes in the selected set. The pruning process ensured that if a node $v$ is pruned from the selection set, the nodes connected to $v$ can 
connect to other dominators. The connectivity of other nodes in the selected sub-network is still keeped, otherwise, the node can not be pruned. On the other hand, unless the nodes that can dominate node $v$ and his neighbor, the nodes will not be checked for replacing node $v$. Thus, the connectivity of the selection set is guranteed.

Besides, the DMPID algorithm is a heuristic algorithm based ob the neighbor and two hops neighbor information of the nodes in the network. The selection of the sub-graph is not optimal in global in terms of the information spread probability and the size of the selection. This is the same case as that most distributed algorithms[3]. In DMPID algorithm, the message passing process include the neighbor and two hops neighbor finding and the pruning procedure. The total information exchanged during these procedure is comparable with the case in most of the distributed algorithms[3]. In cyber physical integrated network, a node's neighbor is bounded due to the hardware restrictions. And the computation complexity on each node is rely on the number of his neighbor, as a result, the complexity of the algorithm is bounded.

\section{EVALUATION}

The DMPID algorithm focuses on finding a sub-network to make the communication probability maximized. The most similar work we found is Lin et. al. [3]. They tried to find a connected dominant set for a cognitive network. The comparable parameters are the size of the selection, and the weight of the selection for maximizing the lifetime or the information spread probability.

\section{A. Evaluation Setup}

The nodes distribution of the network affects the selection of the sub-network heavily. In the evaluation, both the density of the nodes and the probability of the links between nodes are randomly generated. Take the number of the nodes as variable, and observe the variation of the size of the selection and the information spread probability.

In each simulation, we generate the network using a kmeans clustering method to assure the nodes distribution and the density. Meanwhile, keep the network as a connected network. The simulation is taken out in two different scenarios, sparse network and dense network. In sparse network, the links distribute in a probability of $30 \%$, while in the dense network the distribute probability as $75 \%$. Besides, the probability for two objects communication is uniformly distributed. The coefficient for the dominant ability is set as alpha to be 0.7 , and the beta to be 3 . The simulation is run for 50 times and the average result is taken to reduce the influnce of randomness.

\section{B. The Results}

The evaluation is taken out to validate the algorithm's correctness and efficiency in terms of the size of the selection and the maximum information diffusion probability.
1) The size of the selection: In DMPID algorithm, the size of the selection is considered accompany with the information spread probability, which is the weight of the links. As stated in algorithm design, in order to get a higher information spread probability, some additional nodes may be added to the selection, make the size of the selection larger. In Fig. 3, the size of the selection of the DMPID algorithm is a little bit larger. This is because in DMPID algorithm, some intermediate nodes is additionally selected to make the information spread probability higher. Besides, in Fig. 3, the size of the selection is comparable with the L-MLCDS algorithm in the case of sparse network. However, in the case of dense network, the size of the selection is larger than that of L-MLCDS algorithm. The reason for such a result is that when the density of the links in the network reaches $70 \%$, there is more chance the links for a higher information spread probability exists. As a result the intermediate nodes chosen as an additional dominator enlarged the size of the selection. However, in Fig. 3 , we can easily find that the size of the selection is still in an acceptable level compared with the scale of the network.
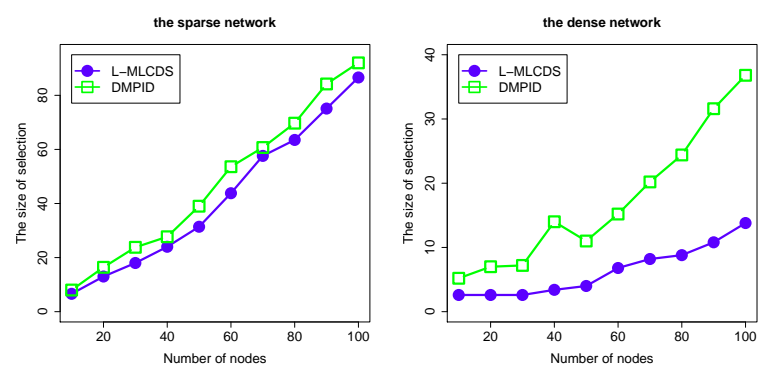

Fig. 3: Simulation result for size of CDS

2) The information spread probability: At any given time, the information spread probability is bounded by the minimum probability link between the objects, which means that two objects in the current time has the lowest probability to communicate with each other than the other neighbor in the target group of objects. The DMPID algorithm tries to find the objects that can communicate at a higher probability, and assure that a object can communicate directly with at least one of the objects in the selection. In Fig. 4, the DMPID algorithm can hardly get a higher information spread probability in sparse network. Because in sparse network, there is few links to an object and the selection can be made only in a few chances. So the choose of intermediate nodes process in the algorithm is ignored in most cases. As a result, the communication links are keeped the same as that in L-MLCDS. Beside, the information diffusion probability in sparse network is lower for that in some cases, the links with small communication probability maybe the sole chose for the object communicate with the objects in the selection. However, in densed network, the choose for intermediate objects may happen in most situations. As a result, the information diffusion probability of the selection is higher in most cases. And in some cases, as shows in Fig. 4, the result of the DMPID algorithm can 
be twice times compared with L-MLCDS in terms of the information diffusion probability.
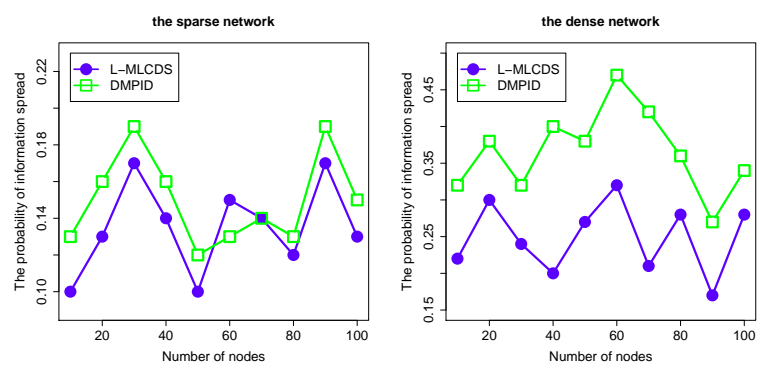

Fig. 4: The state of the network

\section{RELATED WORK}

Information diffusion is a hot topic in social network research. The reserch includes model for the information diffussion in specific form of networks [4], [5], speed up the information diffusion in social networks to get a higher influence [6], [7], explore the diffusion way of some types of information in social network and to control them or direct the information diffusion [8], [9] etc. These work focus on the information diffusion in online network, not considering the physical elements.

There is rarely research for information diffusion in cyberphysical integrated network. [10] explored the impact of the speed and scale of information diffusion by integrating the physical network such as face to face communication, phone calls, and the online social network such as Facebook, Twitter, YouTube etc. [11] studied the information diffusion behavior of real-time information in an overlaying social-physical network. They assume that the physical network consist of many cliques and information can spread quickly in the cliques. However, how these cliques are formed and the information diffusion among cliques are not considered.

Selecting a sub-network in wireless network is an important research topic. Lots of research focused on minimizing the size of the selection and the efficiency of the select process, and they assumed that the topology of the network is known ahead [12]. Distributed selecting a sub-network in wireless network is divided to additional based and prune base methods [13]. But they consider the network links are static. The cyber-physical integrated network is a dynamic network, and information diffusion in such a network must deal with the dynamic. The most similiar work to our's is Lin et. al. [3], they studied the dynamic situation in cognitive network.

\section{CONCLUSION}

This paper focuses on the information diffusion in the cyber physical integrated network. Emphasize the dynamic of the network and the people oriented information spread. In a distributed cyber physical integrated network model, the communication of the objects are a probability event. Basing on the statistic strategy, the information diffusion in such a network is dealt with finding a sub-network to accomplish the task. And the purpose is to make a selection so as the size is minimized and the infomation diffusion probability is maximized over the probability links. By designing DMPID algorithm, the selection is made in a distributed manner and used the prune based distribute algorithm design strategy. Extensive simulation shows that the DMPID algorithm performs well in different distributed networks. The future work include the study on the information diffusion over the cyber physical integrated network from the angle of time migration.

\section{ACKNOWLEDGMENT}

This work is supported by NSFCRGC Joint Research Scheme(No. N_PolyU519/12), and is partially supported by NSFC of the Hunan Province (11JJ7003), NSFC (61070203, 61202484) and Qing Lan Project.

\section{REFERENCES}

[1] Y. Huang, X. Ma, J. Cao, X. Tao, and J. Lu, "Concurrent event detection for asynchronous consistency checking of pervasive context," in Pervasive Computing and Communications, 2009. PerCom 2009. IEEE International Conference on. IEEE, 2009, pp. 1-9.

[2] V. Raychoudhury, J. Cao, W. Zhu, and A. D. Kshemkalyani, "Context map for navigating the physical world," in Parallel, Distributed and Network-Based Processing (PDP), 2012 20th Euromicro International Conference on. IEEE, 2012, pp. 146-153.

[3] Z. Lin, H. Liu, X. Chu, Y.-W. Leung, and I. Stojmenovic, "Constructing connected-dominating-set with maximum lifetime in cognitive radio networks," Computers, IEEE Transactions on, vol. Early Access Articles, no. 1,2013

[4] T. Iwata, A. Shah, and Z. Ghahramani, "Discovering latent influence in online social activities via shared cascade poisson processes," in Proceedings of the 19th ACM SIGKDD international conference on Knowledge discovery and data mining. ACM, 2013, pp. 266-274.

[5] C. Jiang, Y. Chen, and K. R. Liu, "Modeling information diffusion dynamics over social networks," in Acoustics, Speech and Signal Processing (ICASSP), 2014 IEEE International Conference on. IEEE, 2014, pp. 1095-1099.

[6] I. Scholtes, N. Wider, R. Pfitzner, A. Garas, C. J. Tessone, and F. Schweitzer, "Causality-driven slow-down and speed-up of diffusion in non-markovian temporal networks," Nature communications, vol. 5, 2014.

[7] C. Jiang, Y. Chen, and K. R. Liu, "Evolutionary dynamics of information diffusion over social networks," Signal Processing, IEEE Transactions on, vol. 62, no. 17, pp. 4573-4586, 2014.

[8] A. Banerjee, A. G. Chandrasekhar, E. Duflo, and M. O. Jackson, "Gossip: Identifying central individuals in a social network," National Bureau of Economic Research, Tech. Rep., 2014.

[9] A. Runka and T. White, "Towards intelligent control of influence diffusion in social networks," Social Network Analysis and Mining, vol. 5, no. 1, pp. 1-15, 2015.

[10] O. Yağan, D. Qian, J. Zhang, and D. Cochran, "Conjoining speeds up information diffusion in overlaying social-physical networks," Selected Areas in Communications, IEEE Journal on, vol. 31, no. 6, pp. 10381048, 2013.

[11] D. Qian, O. Yağan, L. Yang, J. Zhang, and K. Xing, "Diffusion of real-time information in overlaying social-physical networks: network coupling and clique structure," Networking Science, vol. 3, no. 1-4, pp. 43-53, 2013.

[12] L. Ruan, H. Du, X. Jia, W. Wu, Y. Li, and K.-I. Ko, "A greedy approximation for minimum connected dominating sets," Theoretical Computer Science, vol. 329, no. 1, pp. 325-330, 2004.

[13] I. Stojmenovic, M. Seddigh, and J. Zunic, "Dominating sets and neighbor elimination-based broadcasting algorithms in wireless networks," Parallel and Distributed Systems, IEEE Transactions on, vol. 13, no. 1, pp. 14-25, 2002. 